Original Research Paper

\title{
Treatment Adherence among Diabetes Mellitus Type II Patients at Ambulatory Clinics in the Western Region of Saudi Arabia: Descriptive Correlational Study
}

\author{
Louise de Villiers and Jehad O. Halabi \\ College of Nursing-Jeddah, King Saud bin Abdullaziz University for Health Sciences, Kingdom of Saudi Arabia
}

Article history

Received: 11-01-2015

Revised: 14-07-2015

Accepted: 11-09-2015

Corresponding Author: Louise de Villiers

College of Nursing-Jeddah,

King Saud bin Abdullaziz

University for Health Sciences,

Kingdom of Saudi Arabia

E-mail: 1devilliers58@gmail.com

\begin{abstract}
Diabetes mellitus is a common problem in Saudi Arabia. Adherence to the pharmacological and lifestyle interventions regimens is essential for weight and glycaemic control, as well as prevention of comorbidities. This study described the levels of treatment adherence among diabetes mellitus type II patients, explored the factors which influenced adherence and identified predictors of adherence. It was conducted in five ambulatory care clinics in the Western Region. The accessible population comprised diagnosed diabetes mellitus type II patients who received treatment at the ambulatory clinics. Persons aged $\geq 18$ years and had been placed on medication and lifestyle intervention regimens at least six months prior to data collection, qualified for inclusion. The sample comprised 1,409 randomly selected participants. Participants completed a structured Arabic questionnaire, assisted by research assistants. The research assistants furthermore analysed the participants' clinical records, using a structured checklist. Descriptive and inferential statistics were performed to quantify adherence, explore the relationships among variables and identify the predictors of adherence within the framework of the Health Belief Model. High prevalence of obesity and low prevalence of glycaemic control were found. Participants inadequately adhered to the medication and lifestyle intervention regimens, as well as blood glucose self-monitoring. The findings revealed various perceptual problems which might have contributed to inadequate adherence. The identified predictors of adherence indicated the essential requirements for health care interventions to improve treatment adherence. It is recommended that health education should address sociocultural in addition to biomedical matters to enhance the cultural congruency of interventions and ultimately adherence. Diabetes mellitus management aimed at achieving glycaemic and weight control and protection against comorbidities requires health education, motivation and support targeted at medication and in particular lifestyle adherence.
\end{abstract}

Keywords: Adherence, Descriptive Correlational Research, Diabetes Mellitus, Glycaemic Control, Health Belief Model, Lifestyle Adherence, Medication Adherence, Saudi Arabia

\section{Introduction}

\section{Prevalence and Contributing Factors}

The Kingdom of Saudi Arabia (KSA) has a total population of 27,345,986 (CIA, 2014). The prevalence of Diabetes Mellitus (DM) has reached epidemic proportions affecting an estimated 1.1 million adult $(\geq 15$ years) males and 775,000 females (MOH, 2014). DM and related co-morbidities constitute the leading cause of morbidity, disability and mortality. The estimated agestandardised death rate for DM and cardiovascular disease is 540.6 per 100,000 for males and 347.6 per 100,000 for females (WHO, 2011a).

Inactivity and unhealthy eating patterns are related to obesity and DM (Badran and Laher, 2012; Lawrence et al., 2012). In the KSA, the main behavioural risk factor for chronic disease is physical inactivity and the main metabolic risk factor is overweight, followed by elevated 
cholesterol and obesity (WHO, 2011a). Obesity affects up to $29 \%$ of the population, with a prevalence of $34 \%$ among females and 24\% among males. The 55-64 year age group is especially affected with a prevalence of $48 \%$ (MOH, 2014).

\section{Disease Management and Adherence}

Diabetes management entails pharmacological therapy and lifestyle interventions (Saleh et al., 2014). Oral anti-diabetic agents and meal planning are usually prescribed for type II DM (Smeltzer et al., 2010). Lifestyle interventions include physical activity, a wellbalanced diet, weight loss and self-monitoring (Lawrence et al., 2012).

DM requires lifelong adherence to health regimens. Adherence refers to active, voluntary and collaborative patient involvement to produce a therapeutic effect, which results from internalising treatment recommendations and showing commitment to selfcare behaviour (Delamater, 2006; Kyngäs, 2007).

According to the Health Belief Model, the perceptions of persons suffering from disease would invariably influence their health behaviour. Patients diagnosed with DM would therefore have to consider the disease severity and their vulnerability to develop complications before judging whether the benefits of adherence outweigh encountered barriers. Decisions related to adherence are also influenced by demographic and psychosocial factors, as well as cues to action (Rimer and Glanz, 2005).

Adherence is promoted when patients have willpower, are motivated and have a sense of personal energy. Patients are likely to adhere when the treatment regimen makes sense to them, when it seems effective and when they feel they have the ability to succeed at the regimen. A supportive therapeutic relationship with health care providers together with frequent contact, promote treatment adherence (Delamater, 2006; Kyngäs, 2007). Patients adhere better to medication compared with lifestyle intervention regimens, as well as to simpler compared with complex regimens (Khattab et al., 2010).

Non-adherence indicates failure to consistently apply treatment recommendations independently (Delamater, 2006; Kyngäs, 2007). Non-adherence can occur when a health condition is chronic, when the course of symptoms varies or when symptoms are not apparent. Inappropriate health beliefs and low self-efficacy may also lead to non-adherence (Adisa et al., 2009; Delamater, 2006).

A systematic review study found an average adherence rate of $68 \%$ among diabetics and that almost half of all patients with chronic diseases stopped refilling prescriptions within one year of commencing their treatment (Melko et al., 2010). Research also revealed poor adherence to vascular-protection medication among diabetics $(\mathrm{N}=170,381)$ (Asghari et al., 2010). Nonadherence results in complications, increased treatment costs and reduced quality of life.

\section{Problem Statement}

The researchers observed the occurrence of uncontrolled DM among diabetics in ambulatory care settings in Jeddah, despite numerous health education efforts. Previous research in this context focussed mainly on the prevalence and epidemiology of DM or adherence patterns. Limited information was available about the factors influencing adherence among diabetics.

This study was based on the Health Belief Model. It investigated the levels of treatment adherence among DM type II patients and sought to identify the factors influencing adherence. The following research questions guided the study:

- How adherent are type II diabetics with their prescribed medication and lifestyle intervention regimens?

- How do type II diabetics perceive the Health Belief Model variables of severity, risk, benefits, barriers, intrapersonal factors, health care provider factors and cues to action?

- What are the interrelationships between treatment adherence, socio-demographic factors, HblAc status, weight status; duration of illness and the Health Belief Model variables?

- What are the predictors for treatment adherence?

\section{Materials and Methods}

\section{Design}

This descriptive-correlational study was conducted in five ambulatory care settings of the Ministry of National Guard in the Western Region of the KSA. The accessible population comprised diagnosed DM type II patients who received treatment at the clinics. Persons aged $\geq 18$ years that had been placed on medication and lifestyle intervention regimens at least six months prior to data collection were targeted.

\section{Sample Selection}

A multistage cluster sampling technique was applied. Firstly, five clinics were selected from a sampling frame of eight clinics, using a simple random sampling technique. Secondly, proportional to size sampling was applied to select participants from each of the five selected clinics. The scheduled male and female DM clinic appointments served as separate sampling frames from which a simple random selection was made. The minimum sample size was calculated to be 646 using the sample size formula for single proportions. After accommodating for cluster 
sampling, once-off data collection and a $10 \%$ nonresponse rate, the required sample size was 1,421 .

\section{Data Collection}

This study utilised a structured self-report method involving questionnaire completion. The questionnaire, which had been used in a previous study, was adapted and pretested for this study (Edo and De Villiers, 2012). In addition to this, experts judged the design of the questionnaire, applicability of the items, extent to which the questionnaire reflected the Health Belief Model and the linguistics of the Arabic translation.

Data collection occurred between March 2013 and May 2014. The Arabic questionnaire was administered by ten Arabic speaking, qualified DM care clinicians. These research assistants assisted participants with questionnaire completion. In addition, they measured participants' weight and height, documented their HbA1c values and extracted data from the participants' clinical records using a structured observational checklist.

Participants' responses to biographical items were captured using scales supporting nominal and ordinal levels of measurement. Age, number of dosages skipped and number of cigarettes smoked were measured at the ratio level. The sections which measured adherence and Health Belief Model variables included 4-point Likert-type scales.

\section{Data Management and Analysis}

Data analysis was done using SPSS Version 20. Entered data was scrutinised for missing data, outliers and wild codes. Time series data transformations were used to predict the values of the missing data. All the variables were subjected to descriptive analysis. Factor analysis was done to identify clusters of variables which were most closely linked together (Polit and Beck, 2012). Where appropriate the variables which emerged from the factor analysis were also subjected to statistical analysis.

Interrelationships and group differences were investigated using the chi-square and one-way ANOVA techniques. Stepwise multiple linear regression analysis was used to identify the predictor variables for adherence (Polit and Beck, 2012). The significance level was $\mathrm{p}<0.05$.

Responses to biographical and Likert-type scale items were collapsed into two groups in order to conduct the chi-square calculations as indicated in Tables 2-5. In order to apply the ANOVA technique, responses to the item related to level of education, were collapsed into three groups, namely no schooling, partial schooling and high school certificate and above.

\section{Research Ethics}

Approval was obtained from the King Abdullah International Medical Research Centre, the Director of
Primary Health Care Services and participants. The participants were assured that non-participation, withdrawal or their responses would not result in prejudicial treatment from the research team or the clinic staff. Privacy was maintained and extra precautions were taken to ensure that participation by females occurred in accordance with cultural requirements.

The main goal of any research is to generate sound scientific knowledge through honest methods (Polit and Beck, 2012). This study was conducted as outlined in the approved research proposal and the researchers' interpretations were statistically supported.

\section{Results}

\section{Biographical Results}

The sample size was 1,409 including 680 (48\%) males and $729(52 \%)$ females. The mean age was $55( \pm 11.06)$ years. A total of $702(50 \%)$ had no schooling, 532 (38\%) had partial schooling and $175(12 \%)$ achieved a high school certificate and above. More than half of the participants $(\mathrm{n}=784 ; 56 \%)$ were diagnosed $>5$ years previously.

\section{Diabetic History}

The diabetic history of participants is indicated in Table 1. Glycaemic control was measured using HbA1c results based on laboratory analysis of three millilitres of blood submitted in an EDTA tube (lavender top). Poor glycaemic control was defined as HbAlc $\geq 7 \%$ (Al Hayek et al., 2013). Most participants $(n=1,095 ; 78 \%)$ presented with poor glycaemic control $(\bar{x}=8.64, \pm 1.93)$.

BMI was calculated using the formula BMI=weight $(\mathrm{kg}) /[\text { height }(\mathrm{m})]^{2}$. The mean BMI was $31.49( \pm 5.62)$. Most participants were obese $(n=817$; $58 \%)$ and $456(32 \%)$ were overweight. Females were predominately obese whereas males were almost equally obese and overweight.

The most frequently reported complications were hypertension $(n=593 ; 42 \%)$ and bad eyesight $(n=401$; $28 \%$ ). Participants' medical records included evidence of coronary artery disease $(\mathrm{n}=232 ; 16 \%)$ and retinopathy $(\mathrm{n}=197 ; 14 \%)$.

Very few participants reported taking over-thecounter medication and traditional herbs. Participants reported taking anti-diabetics $(\mathrm{n}=906 ; 64 \%)$ and insulin $(n=195 ; 14 \%)$. This was inconsistent with the medical records, which indicated that $1,253(89 \%)$ of participants were on anti-diabetics and 492 (35\%) were on insulin. There was consistency between the participants' responses $(n=369 ; 26 \%)$ and their medical records $(n=361 ; 25 \%)$ with regard to being on medication to treat complications. 
Table 1. Diabetic history $(\mathrm{n}=1409)$

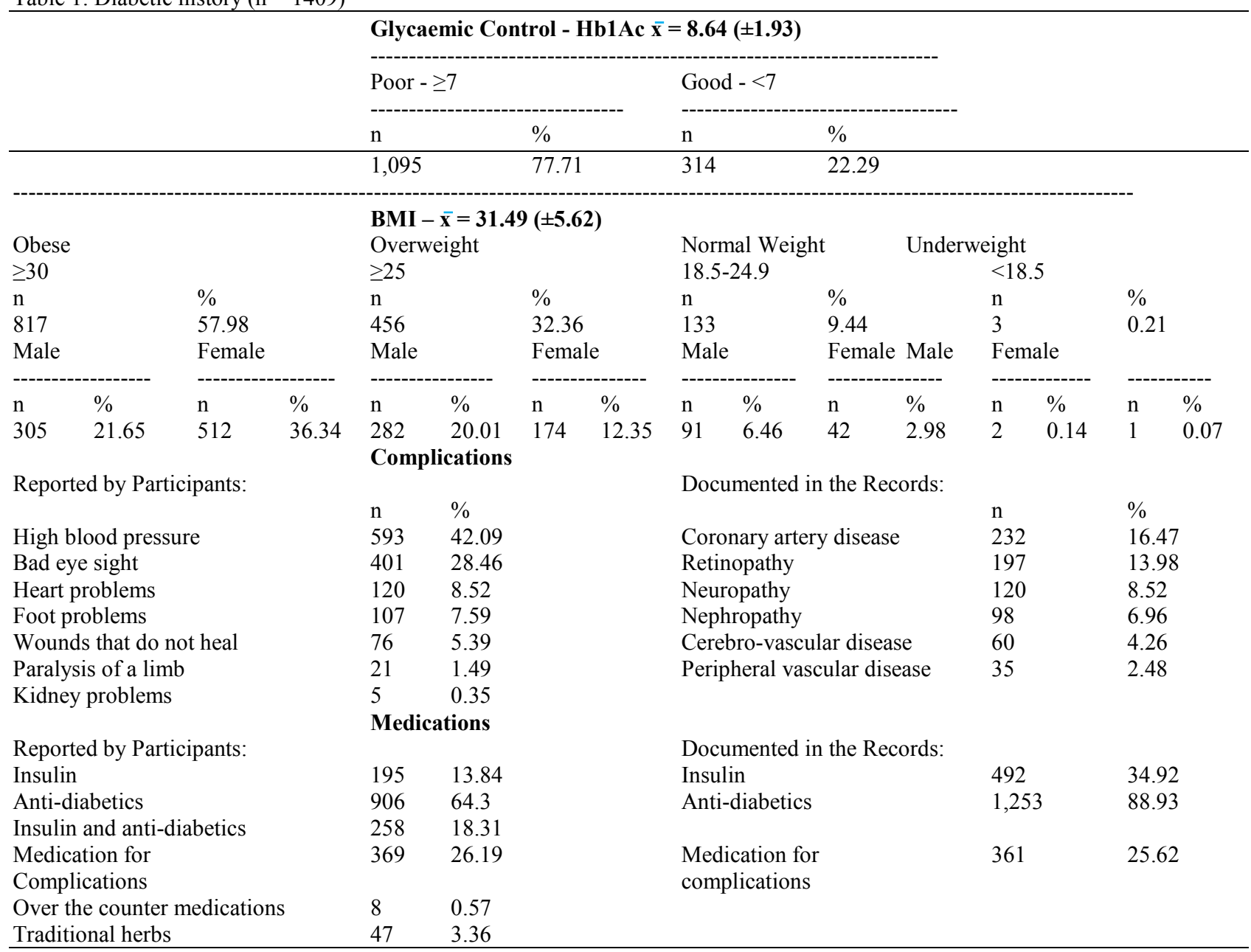

WHO (2011b)

\section{Treatment Adherence}

Adherence to the medication and lifestyle interventions regimens were investigated. The adherence results are summarised in Table 2. Participants reported being medication adherent on the medication adherence scale. However, almost half $(\mathrm{n}=666 ; 47 \%)$ failed to monitor their blood glucose levels more than once a week and approximately a third $(\mathrm{n}=448 ; 32 \%)$ reported that they skipped dosages during the past three days.

Participants reported adherence to most of the required lifestyle choices as indicated in Table 2. However, they consumed carbohydrates like bread, pastry and rice daily and animal protein (chicken and meat) several times a week, while exercise occurred only about once a week. Participants furthermore reported that they consumed fruit and vegetables at least several times a week.

\section{Perception of Severity, Risk, Benefits and Barriers}

Results pertaining to perception are summarised in Table 3. Most participants regarded DM as a "serious" or "very serious" condition. However, it is noteworthy that
$563(40 \%)$ participants indicated that their condition was "not at all" or "somewhat" serious. Participants reported that they had at least $50 \%$ risk to develop visual problems. This was followed by $25 \%-49 \%$ risk to develop diabetic coma, heart problems, kidney problems and stroke. It is noteworthy that participants rated the risk for visual complications higher than cardiovascular complications, despite the higher prevalence of cardiovascular complications as previously discussed (Table 1).

Participants considered treatment adherence as highly beneficial. However, they encountered several barriers which made it difficult to adhere. Problematic were ineffectiveness of their medication and lack of discipline to follow their diet or stop smoking. Somewhat problematic were forgetfulness to obtain a refill proactively, numerous lifestyle changes, cultural restrictions against declining food, as well as lack of time and facilities to exercise. While $372(26 \%)$ participants experienced difficulties in honouring clinic appointments, evidence of follow-up visit non-adherence was found in $463(33 \%)$ medical records. 
Table 2. Treatment Adherence $(n=1409)$

\begin{tabular}{|c|c|c|c|c|c|c|}
\hline & \multirow[b]{2}{*}{ Mdn } & \multirow[b]{2}{*}{ IQR } & \multicolumn{2}{|c|}{$\begin{array}{l}\text { Daily or several } \\
\text { times a week }\end{array}$} & \multicolumn{2}{|c|}{$\begin{array}{l}\text { Once a week } \\
\text { or less }\end{array}$} \\
\hline & & & $\mathrm{n}$ & $\%$ & $\mathrm{n}$ & $\%$ \\
\hline & & & \multicolumn{4}{|c|}{ Medication Adherence $(\alpha=0.79)$} \\
\hline \multicolumn{7}{|l|}{ Non-adherence-intrapersonal reasons: } \\
\hline Try traditional herbs ${ }^{\mathrm{a}}$ & 4 & 0 & 52 & 3.69 & 1,357 & 96.31 \\
\hline Feeling better ${ }^{\mathrm{a}}$ & 4 & 0 & 197 & 13.98 & 1,212 & 86.02 \\
\hline Forgetfulness $^{\mathrm{a}}$ & 4 & 1 & 226 & 16.04 & 1,183 & 83.96 \\
\hline \multicolumn{7}{|l|}{ Non-adherence-medication related reasons: } \\
\hline Frequently change of medication $^{\mathrm{a}}$ & 4 & 0 & 192 & 13.63 & 1,217 & 86.37 \\
\hline Ineffective medication $^{\mathrm{a}}$ & 4 & 0 & 220 & 15.61 & 1,189 & 84.39 \\
\hline Fear negative side-effects* & 4 & 0 & 214 & 15.19 & 1,195 & 84.81 \\
\hline Feeling worse due to medication ${ }^{a}$ & 4 & 0 & 281 & 19.94 & 1,128 & 80.06 \\
\hline \multicolumn{7}{|l|}{ Specific adherence behaviour: } \\
\hline Taking medication as prescribed & 4 & 0 & 1,335 & 94.75 & 74 & 5.250 \\
\hline \multirow[t]{2}{*}{ Checking blood glucose levels } & 2 & 2 & 743 & 52.73 & 666 & 47.27 \\
\hline & & & \multicolumn{4}{|c|}{ Lifestyle Adherence $(\alpha=0.60)$} \\
\hline \multicolumn{7}{|l|}{ Healthy nutritional choices: } \\
\hline Eating vegetables & 3 & 1 & 1,202 & 85.31 & 207 & 14.69 \\
\hline Eating fruits & 3 & 1 & 1,085 & 77 & 324 & 23 \\
\hline \multicolumn{7}{|l|}{ Well-being supporting choices: } \\
\hline Sufficient sleep & 4 & 1 & 1,228 & 87.15 & 181 & 12.85 \\
\hline Relaxation & 4 & 1 & 1,143 & 81.12 & 266 & 18.88 \\
\hline Sufficient water intake & 4 & 1 & 1,185 & 84.1 & 224 & 15.90 \\
\hline Physical exercise of at least 30 minutes & 2 & 2 & 439 & 31.16 & 970 & 68.84 \\
\hline \multicolumn{7}{|l|}{ Unhealthy nutritional choices: } \\
\hline Eating fast food ${ }^{\mathrm{a}}$ & 4 & 1 & 212 & 15.05 & 1,197 & 84.95 \\
\hline Drinking soft drinks ${ }^{\mathrm{a}}$ & 4 & 1 & 326 & 23.14 & 1,083 & 76.86 \\
\hline Eat foodstuffs like cake or chocolate ${ }^{a}$ & 4 & 1 & 317 & 22.50 & 1,092 & 77.50 \\
\hline Eat foodstuffs like meat or, chicken ${ }^{a}$ & 2 & 1 & 1,286 & 91.27 & 123 & 8.730 \\
\hline Eat foodstuffs like bread, pastry or rice & 1 & 1 & 1,187 & 84.24 & 222 & 15.76 \\
\hline
\end{tabular}

${ }^{\mathrm{a}}$ Reverse coding

\section{Intrapersonal Factors, Health Care Provider Factors and Cues to Action}

The results related to this section are summarised in Table 4. Participants understood the nature of DM, symptoms of abnormal blood glucose levels and causes of blood glucose fluctuations better than the effect of their medications, importance of adherence, ways of keeping blood glucose under control and the meaning of blood glucose readings. They strongly believed that their physical health was determined by the will of God rather their own actions. A majority reported a shared responsibility with the doctor to manage their condition and ability to cope at home. It is noteworthy that $1,231(87 \%)$ of participants rejected a belief that DM is curable with traditional herbs. This corresponded with the finding that 1,362 (96\%) did not use traditional herbs frequently (Table 2).

Participants perceived the stated health care provider factors highly positively. It is noteworthy that they viewed the waiting time in the clinic less favourably compared to aspects related to the health care team and health care received.
Regarding cues to action, participants indicated that they were highly motivated by advice given by doctors and health educators. They were also motivated by advice given by family and other diabetics, text messages from the clinic and TV programmes on DM. Less motivating were friends' advice, radio programmes, posters and leaflets, as well as the death of a relative or friend. Written articles and information obtained from the Internet were not motivational.

\section{Interrelationships between the Research Variables}

Table 5 indicates significant relationships between the research variables. Female participants and participants diagnosed $>5$ years previously were significantly more likely to report medication adherence than their counterparts $(p=<0.001)$. Participants with no schooling were most medication adherent and those with partial schooling were significantly less adherent $(F(2)=8.34 ; p=<0.001)$. Medication adherent participants were significantly more likely to present with $\mathrm{BMI} \geq 25$ than nonadherent participants $(\mathrm{p}=0.007)$. 
Table 3. Perceptions of Severity, Risk, Benefits and Barriers $(n=1409)$

\begin{tabular}{|c|c|c|c|c|c|c|}
\hline & Mdn & IQR & $\mathrm{n}$ & $\%$ & $\mathrm{n}$ & $\%$ \\
\hline & \multicolumn{6}{|c|}{ Perception of Severity } \\
\hline & & & \multicolumn{2}{|c|}{ Very serious/Serious } & \multicolumn{2}{|c|}{ Maybe/Not at all serious } \\
\hline \multirow[t]{3}{*}{ Seriousness of DM } & 3 & 2 & 846 & 60 & 563 & 40 \\
\hline & \multicolumn{6}{|c|}{ Perception of Risk $(\alpha=0.95)$} \\
\hline & & & \multicolumn{2}{|c|}{$50-100 \%$ Chance } & \multicolumn{2}{|c|}{$0-49 \%$ Chance } \\
\hline \multicolumn{7}{|l|}{ Physical problems: } \\
\hline Bad eye sight & 3 & 2 & 755 & 53.58 & 654 & 46.42 \\
\hline Diabetic coma & 2 & 3 & 527 & 37.4 & 882 & 62.6 \\
\hline Heart problems & 2 & 2 & 566 & 40.17 & 843 & 59.83 \\
\hline Kidney problems & 2 & 2 & 537 & 38.11 & 872 & 61.89 \\
\hline Stroke & 2 & 2 & 459 & 32.58 & 950 & 67.42 \\
\hline Amputation & 1 & 1 & 412 & 29.24 & 997 & 70.76 \\
\hline Disrupted eating habits & 1 & 2 & 461 & 32.72 & 948 & 67.28 \\
\hline \multicolumn{7}{|l|}{ Psycho-social problems: } \\
\hline Marital & 1 & 1 & 273 & 19.38 & 1,136 & 80.62 \\
\hline Lower quality of life & 1 & 1 & 229 & 16.25 & 1,180 & 83.75 \\
\hline Emotional & 1 & 1 & 207 & 14.69 & 1,202 & 85.31 \\
\hline Disrupted family life & 1 & 1 & 196 & 13.91 & 1,213 & 86.09 \\
\hline Disrupted social life & 1 & 1 & 199 & 14.12 & 1,210 & 85.88 \\
\hline Burden on family & 1 & 1 & 193 & 13.7 & 1,216 & 86.3 \\
\hline Disrupted work life & 1 & 1 & 188 & 13.34 & 1,221 & 86.66 \\
\hline Disrupted education & 1 & 1 & 178 & 12.63 & 1,231 & 87.37 \\
\hline \multirow[t]{3}{*}{ Financial } & 1 & 0 & 153 & 10.86 & 1,256 & 89.14 \\
\hline & \multicolumn{6}{|c|}{ Perception of Benefits $(\alpha=\mathbf{0 . 9 3})$} \\
\hline & & & \multicolumn{2}{|c|}{ Beneficial/very beneficial } & \multicolumn{2}{|c|}{ Somewhat/not at all beneficial } \\
\hline Glucose control & 4 & 1 & 1,388 & 98.51 & 21 & 1.49 \\
\hline Reduced hospital/clinic visits & 4 & 1 & 1,352 & 95.95 & 57 & 4.05 \\
\hline Protection from complications & 4 & 1 & 1,333 & 94.61 & 76 & 5.39 \\
\hline Quality of life & 4 & 1 & 1,352 & 95.95 & 57 & 4.05 \\
\hline Well-being & 4 & 1 & 1,344 & 95.39 & 65 & 4.61 \\
\hline Reduced expenses & 4 & 1 & 1,269 & 90.06 & 140 & 9.94 \\
\hline Peace of mind & 4 & 1 & 1,269 & 90.06 & 140 & 9.94 \\
\hline \multirow{3}{*}{ Longevity } & 4 & 1 & 1,203 & 85.38 & 206 & 14.62 \\
\hline & \multicolumn{6}{|c|}{ Perception of Barriers $(\alpha=0.87)$} \\
\hline & & & \multicolumn{2}{|c|}{$\begin{array}{l}\text { Problematic/Extremely } \\
\text { problematic }\end{array}$} & \multicolumn{2}{|c|}{$\begin{array}{l}\text { Not at all/Somewhat } \\
\text { problematic }\end{array}$} \\
\hline \multicolumn{7}{|l|}{ Treatment related: } \\
\hline Ineffectiveness of medicine & 3 & 3 & 820 & 58.2 & 589 & 41.8 \\
\hline Forgetting to obtain refill & 2 & 2 & 538 & 38.18 & 871 & 61.82 \\
\hline Numerous lifestyle changes & 2 & 2 & 397 & 28.18 & 1,012 & 71.82 \\
\hline \multicolumn{7}{|l|}{ Intrapersonal: } \\
\hline Lack of discipline & 3 & 3 & 711 & 50.46 & 698 & 49.54 \\
\hline Cultural restrictions (declining food) & 2 & 2 & 602 & 42.73 & 807 & 57.27 \\
\hline Lack of time to exercise & 2 & 2 & 517 & 36.69 & 892 & 63.3 \\
\hline Restricted access to area (exercise) & 2 & 2 & 492 & 34.92 & 917 & 65.08 \\
\hline Difficulties - honouring appointments & 2 & 2 & 372 & 26.4 & 1,037 & 73.6 \\
\hline Lack of motivation & 1 & 1 & 301 & 21.36 & 1,108 & 78.63 \\
\hline \multicolumn{7}{|l|}{ Socio-economic obstacles: } \\
\hline Transport & 1 & 1 & 307 & 21.79 & 1,102 & 78.21 \\
\hline Avoid being reminded of illness & 1 & 1 & 277 & 19.66 & 1,132 & 80.34 \\
\hline Social support & 1 & 1 & 238 & 16.89 & 1,171 & 83.11 \\
\hline Finances & 1 & 0 & 118 & 8.37 & 1,291 & 91.63 \\
\hline
\end{tabular}

Males were significantly more likely to report lifestyle adherence than females $(\mathrm{p}=<0.001)$. Participants with partial schooling were significantly more lifestyle adherent than those with no schooling and those with a high school certificate and above $(\mathrm{F}(2)=14.90 ; \mathrm{p}=<0.001)$.
Participants who acknowledged the severity of DM were significantly more likely to report lifestyle nonadherence $(\mathrm{p}=<0.001)$ and present with $\mathrm{Hb} 1 \mathrm{Ac} \geq 7$ ( $\mathrm{p}=$ $0.015)$ than those who underestimated severity. Similarly, participants who were unmotivated by external cues $(\mathrm{p}=$ 0.024 ) had a significantly higher prevalence of $\mathrm{Hb} 1 \mathrm{Ac} \geq 7$ than their counterparts. 
Table 4. Intrapersonal and Health Care Provider Factors and Cues to Action $(n=1409)$

$\begin{array}{lll}\text { Mdn } & \text { IQR } & \mathrm{n} \\ & & \text { SA/Agreed }\end{array}$

$\% \quad \mathrm{n} \quad \%$

$\mathrm{SD} /$ Disagreed

Understanding:

What DM is

Causes of glucose fluctuations

Symptoms of abnormal glucose levels

Importance of adherence

Ways of keeping glucose under control

Effect of medications

Meaning of glucose readings

Self-efficacy:

Able to cope at home

Personal responsibility to manage DM

Locus of control:

Will of God

Doctor primarily responsible to manage DM

Belief:

DM curable with traditional herbs

Respectful treatment - doctor

Respectful treatment - other health care workers

Confidence in health care team.

Competence of health care team.

Health care team understanding concerns.

Usefulness of health education

Health care team listening to concerns.

Effectiveness of issued medicines

Acceptable waiting time at clinic.

Health care providers' advice:

Doctor's advice

Health educator's advice

Social cues:

Advice from other diabetics

Friends' advice

Health education media:

Text messages sent by the clinic

TV programmes on DM

Radio programmes on DM

Posters displayed in the clinic

Health education leaflets

Other cues:

Death of relative/friend due to DM

Newspaper or journal articles

Information on the internet

\section{Intrapersonal Factors $(\alpha=0.81)$}

$\begin{array}{llll}1,376 & 97.66 & 33 & 2.34 \\ 1,364 & 96.81 & 45 & 3.19 \\ 1,220 & 86.59 & 189 & 13.41 \\ 1,379 & 97.87 & 30 & 2.13 \\ 1,368 & 97.09 & 41 & 2.91 \\ 1,304 & 92.55 & 105 & 7.45 \\ 1,220 & 86.59 & 189 & 13.41 \\ & & & \\ 1,199 & 85.1 & 210 & 14.9 \\ 1,121 & 79.56 & 288 & 20.44 \\ 1,121 & & & \\ 1,354 & 79.56 & 288 & 20.44 \\ & 96.1 & 55 & 3.9 \\ 178 & & & 87.37\end{array}$

Health Care Provider Factors $(\alpha=0.95)$

Advice from a family member

SA/Agreed

$\begin{array}{ll}1,406 & 99.79 \\ 1,403 & 99.57 \\ 1,403 & 99.57 \\ 1,402 & 99.5 \\ 1,401 & 99.43 \\ 1,399 & 99.29 \\ 1,400 & 99.36 \\ 1,394 & 98.94 \\ 1,214 & 86.16\end{array}$

Cues to Action $(\alpha=0.90)$

Will definitely/

May motivate

\begin{tabular}{llll}
1,386 & 98.37 & 23 & 1.63 \\
1,377 & 97.73 & 32 & 2.27 \\
1,031 & 73.17 & 378 & 26.83 \\
1,003 & 71.19 & 406 & 28.81 \\
879 & 62.38 & 530 & 37.62 \\
& & & \\
1,031 & 73.17 & 378 & 26.83 \\
1,012 & 71.82 & 397 & 28.18 \\
941 & 66.78 & 468 & 33.22 \\
843 & 59.83 & 566 & 40.17 \\
790 & 56.07 & 619 & 43.93 \\
& & & \\
813 & 57.7 & 596 & 42.3 \\
505 & 35.84 & 904 & 64.16 \\
445 & 31.58 & 964 & 68.42 \\
\hline
\end{tabular}

Participants who acknowledged the severity of DM were significantly more likely to report low perception of risk $(p=<0.001)$ and barriers $(p=<0.001)$ than those who underestimated severity.

It is noteworthy that participants diagnosed $>5$ years ago, participants aged $\geq 55$ years and participants who acknowledged the severity of DM were significantly more likely to report that they were unmotivated by external cues than their counterparts $(\mathrm{p}=<0.001)$.

\section{Predictors of Treatment Adherence}

Predictors of medication adherence were external locus of control and high perception of benefits. Predictors of lifestyle adherence were low perception of barriers and receptiveness to external cues (Table 6). 
Table 5. Interrelationships between the Research Variables $(\mathrm{n}=1409)$

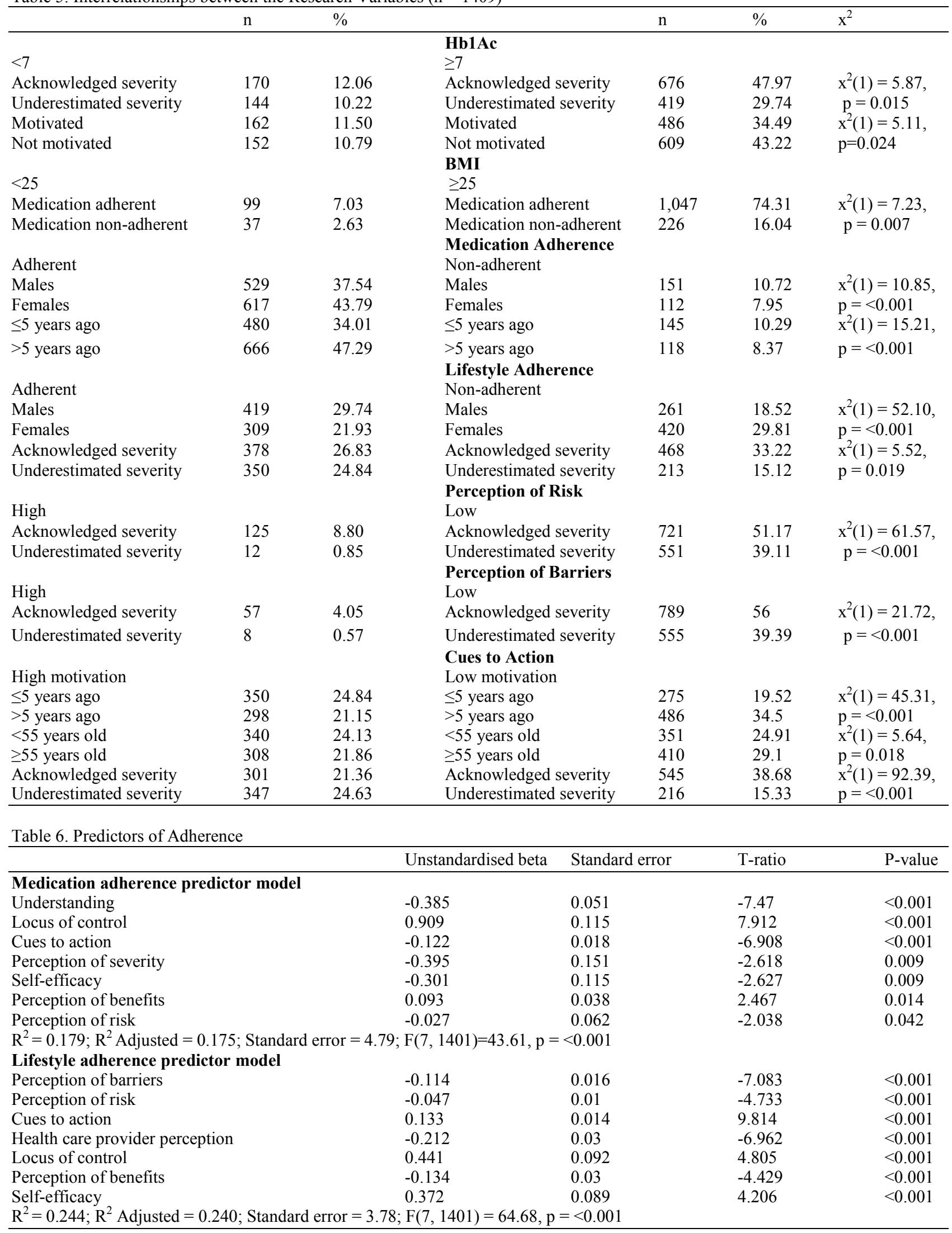




\section{Discussion}

Participants reported adequate medication adherence on the medication adherence scale. However, the discrepancy between the medical records and participants' accounts of their prescribed medication, as well as evidence of skipped dosages, suggested that participants subjectively overestimated their level of medication adherence. The lower adherence among male participants was consistent with previous research results in Nigeria (Adisa et al., 2009) and China (Wong et al., 2011). In contrast, gender did not affect medication adherence in France (Tiv et al., 2012).

This study revealed inadequate lifestyle adherence. Participants frequently consumed animal protein and carbohydrates, failed to exercise regularly and presented with lack of glycaemic and weight control. These results were consistent with previous research, which revealed low adherence to the prescribed eating plan and exercise regimen and a sedentary lifestyle among Saudi participants (Badran and Laher, 2012). Midhet et al. (2010) confirmed that unhealthy diet and physical inactivity are the most important risk factors of type II DM in the KSA. Diet and exercise are essential for blood glucose control and uncontrolled DM can lead to various complications (Al-Hayek et al., 2012).

Both genders showed inadequate lifestyle adherence. However, female participants showed significantly lower adherence levels and a relatively higher perception of barriers. This could be culturally rooted. The literature indicates that cultural barriers, limited access to exercise facilities and easy access to migrant labour encourage a sedentary lifestyle among women in the Gulf Region (Badran and Laher, 2012).

Poor glycaemic control was evident in $78 \%$ of participants. This figure was higher compared to previous research in the KSA. Al-Hayek et al. (2012) found that $71 \% \quad(\mathrm{~N}=147)$ of participants lacked glycaemic control, while Al-Elq (2009) reported a prevalence of $68 \%(\mathrm{~N}=353)$. Systematic review research indicated that glycaemic control was achieved by less than $50 \%$ of type II diabetics due to inadequate adherence to dietary, exercise and medication regimens (García-Pèrez et al., 2013).

Only $53 \%$ of participants monitored their blood glucose levels regularly. This is problematic since selfmonitoring improves blood glucose management and quality of life (Al-Shahrani and Al-Khaldi, 2013). Previous research in the KSA revealed lower levels of self-monitoring adherence, namely 39\% (Al-Hayek et al., 2012) and 30\% (Al-Elq, 2009). Research in Nigeria indicated that inadequate adherence to glucose selfmonitoring could be attributed to lack of knowledge (Adisa et al., 2009).
Adequate consumption of fruit and vegetables, as well as physical exercise, is essential for weight control and prevention of disease (WHO, 2011b). However, the diet in KSA typically includes Kabsa (a meal containing rice and meat), French fries and bakery items. Previous research found an adjusted odds ratio of 5.5 for eating Kabsa and 0.4 for vegetables (Midhet et al., 2010). Participants in this current research consumed fruit and vegetables and meat or chicken several times a week. Bread, pastries or rice were consumed on a daily basis. This frequent intake of carbohydrates could have contributed to the inadequate glycaemic and weight control.

Participants in this study were typically overweight or obese. The mean BMI of 31.49 was consistent with previous research in Riyadh $(\mathrm{N}=113)$, which revealed a mean of 31.06 (Al Hayek et al., 2013) among diabetics. Research in the Asir Region $(\mathrm{N}=14,252)$, obtained a lower BMI, namely a median of 29 (AlShahrani and Al-Khaldi, 2013).

Diabetes, hypertension, overweight and obesity increase the risk of cardiovascular disease. Furthermore, diabetes is one of the leading causes of visual impairment and blindness (WHO, 2014). This study revealed the presence of particularly hypertension, coronary artery disease and retinopathy, as well as the corresponding use of insulin and medication to treat complications. Previous research confirmed that DM is a significant predictor of hypertension in the KSA (Saeed et al., 2011). Badran and Laher (2012) found $31 \%$ prevalence of retinopathy among type II diabetics in Arab speaking countries $(\mathrm{N}=4,758)$. Similarly, systematic review research confirmed the occurrence macro- and micro-vascular conditions as a result of poor glycaemic control (García-Pèrez et al., 2013).

Diabetic patients develop complications as a result of poor insight of the disease and inadequate glycaemic control (Al Hayek et al., 2013). Participants in this research showed partial understanding of DM management, low perception of risk and external locus of control. This might have resulted in inadequate treatment adherence despite appreciating the seriousness of DM and benefits of adherence.

Lack of discipline might have contributed to inadequate lifestyle adherence, inadequate diabetic control and a consequent belief that the medication was ineffective. This latter belief might have further strengthened participants' non-adherence behaviour. Highly educated participants were least lifestyle adherent and therefore lack of discipline is a plausible explanation for the inadequate lifestyle adherence. The literature confirms this conclusion (Delamater, 2006).

Participants indicated that advice by the doctor or health educator would most likely motivate them to adhere. This applied particularly to females, long-time sufferers and older participants. Noteworthy was the low 
motivational value of health education leaflets and posters, printed articles and information on the internet. This latter finding was not surprising considering the low educational status of participants. Research done in Seychelles also highlighted the high motivational value of professional advice and relatively low motivational value of leaflets and posters. Participants in this previous study were however more receptive to health education programmes on television or the radio than the participants in the current study (Edo and De Villiers, 2012).

\section{Conclusion}

This study concludes that treatment adherence was inadequate despite participants' appreciation for the benefits of adherence. High perception of severity and receptiveness to external cues did not necessarily result in behavioural change for glycaemic and weight control. This could be attributed to a low perception of risk to develop complications, barriers which made it difficult to adhere, lack of discipline and insufficient understanding of the disease management requirements. DM management requires culturally congruent health education, motivation and support targeted at medication and in particular lifestyle adherence. The findings of this study can be used to develop effective adherence promotion strategies.

Previous research found that health education is positively associated with improved medication adherence, lifestyle adherence and glycaemic control (Al Hayek et al., 2013). This study recommends that preference should be given to face-to-face health education by doctors and nurse health educators. Television monitors could be utilised in waiting areas to convey health education messages especially targeted at lifestyle adherence. Posters and pamphlets can be used to strengthen health education but should not be regarded as the main means of information dissemination.

Health care professionals should refrain from assuming that diabetics who acknowledge the severity of DM will adhere to treatment. Health education should equally be targeted at diabetics who acknowledge and underestimate the severity of their condition.

It is necessary to address diabetics' cultural views on health, illness and care. All variables of the Health Belief Model should be incorporated in holistic health assessment and education strategies. Health education should address psycho-social topics, including diabetics' religious views, in addition to biomedical aspects related to DM.

The benefits of adherence should be stressed during health education aimed at promoting medication adherence in particular. Health educators should ensure that diabetics fully understand the effects of their medications, their responsibilities regarding blood glucose management, importance of self-monitoring and the meaning of blood glucose values.

Health education should stress the importance of lifestyle adherence in order to enhance the effectiveness of medication. Particular attention should be given to the variety of sources of protein and carbohydrates, as well as how to balance protein and carbohydrate intake for optimal diabetic control. In addition, wellness clinics could be established to promote selfdiscipline and an active lifestyle. Research in Riyadh confirmed that diabetes self-management education improved dietary, exercise and self-monitoring adherence (Al Hayek et al., 2013).

To promote lifestyle adherence in particular, diabetics should be assisted to develop confidence in their ability to exercise self-discipline. Continuous monitoring and support are required to ensure that behavioural changes ensue. Clinics can use mobile devices to send adherence promoting reminders to diabetics. Research by Leon et al. (2015) indicated that adherence support by SMS messages benefitted patients, particularly those who experienced life stressors and those who struggled with adherence.

Research in Mexico by Gutiérrez Herrera et al. (2015) indicated the effectiveness of a health education model which emphasises interactive educational techniques stimulating patient cooperation. The results of this current study suggest that support groups consisting of especially fellow diabetics, family members and to a lesser extent friends would enable diabetics to obtain advice and assistance, overcome barriers and better adhere to the treatment regimen. Participants who suffered from DM for longer than five years can serve as medication adherent role models and resource persons for newly diagnosed diabetics.

A limitation of the study was the under-reporting of smoking behaviour. Cultural factors may have contributed to this. The researchers however believe that this study succeeded in explicating the factors which influence treatment adherence and how treatment adherence could be promoted in the Western region of Saudi Arabia. Replication of the study in other regions of the country is recommended.

\section{Acknowledgement}

The researchers acknowledge the contribution by managers of the five health centres that facilitated access to participants. The research assistants rendered valuable support by collecting data and assisting participants.

\section{Funding Information}

The study was supported by a grant from the King Abdullah International Medical Research Center at King Saud bin Abdulaziz University for Health Sciences, Ministry of National Guard (RJ12/240/J). 


\section{Author's Contributions}

Both authors contributed equally in the preparation, development and publication of this manuscript.

Louise de Villiers: Initiated the study and took primary responsibility for writing the research proposal, seeking funding, implementing the study, corresponding with the funding agency and writing the manuscript.

Jehad O Halabi: Made substantial contributions towards writing the research proposal and manuscript, as well as implementing the study. Took primary responsibility for translating the tool into Arabic, ensuring that the items were relevant to DM in the region and liaising with the research assistants.

\section{Ethics}

No ethical issues are expected to arise after the publication of this manuscript.

\section{References}

Adisa, R., M.B. Alutundu and T.O. Fakeye, 2009. Factors contributing to non-adherence to oral hypoglysemic medications among ambulatory type 2 diabetes patients in South-Western Nigeria. Pharmacy Prac., 7: 163-169.

Al-Elq, A.H., 2009. Current practice in the management of patients with type 2 diabetes mellitus in Saudi Arabia. Saudi Med. J., 30: 1551-1556.

Al Hayek, A.A., A.A. Robert, M.A. Al Dawish, M.M. Zamzami and A.E. Sam et al., 2013. Impact of an education program on patient anxiety, depression, glycemic control and adherence to self-care and medication in Type 2 diabetes. J. Family Community Med., 20: 77-82. DOI: 10.4103/2230-8229.114766

Al-Hayek, A.A., A.A. Robert, A.A. Alzaid, H.M. Nusair and N.S. Zbaidi et al., 2012. Association between diabetes self-care, medication adherence, anxiety, depression and glycemic control in type 2 diabetes. Saudi Med. J., 33: 681-683.

Al-Shahrani, A.M. and Y.M. Al-Khaldi, 2013. Obesity among diabetic and hypertensive patients in Aseer region, Saudi Arabia. Saudi J. Obesity, 1: 14-17. DOI: $10.4103 / 2347-2618.119470$

Asghari, S., J. Courteau, C. Drouin, J.P. Gregoire and A.C. Carpentier et al., 2010. Adherence to vascular protection drugs in diabetic patients in Quebec: A population-based analysis. Diabetes Vascular Dis. Res., 7: 167-171. DOI: 10.1177/1479164109360593

Badran, M. and I. Laher, 2012. Type II diabetes mellitus in Arabic-speaking countries. Int. J. Endocrinol., 1: 11-11. DOI: $10.1155 / 2012 / 902873$

CIA, 2014. The world fact book. CIA.

Delamater, A.M., 2006. Improving patient adherence. Clinical Diabetes, 24: 71-71.

DOI: $10.2337 /$ diaclin.24.2.71
Edo, T.A. and L. De Villiers, 2012. Compliance with anti-hypertensive drug treatment and lifestyle modifications among hypertensive patients on Praslin Island, Seychelles. Africa J. Nursing Midwifery 14: 3-17.

García-Pèrez, L.E., M. Alvarez, T. Dilla, V. Gil-Guillèn and D. Orozco-Beltrán, 2013. Adherence to therapies in patients with type 2 diabetes. Diabetes Ther, 4: 175-194. DOI: 10.1007/s13300-013-0034-y

Gutiérrez Herrera, V.R., H.M. Zerón and M.R. Mendieta Alcántara, 2015. Adherence to two methods of education and metabolic control in type 2 diabetics. Ethiop J. Health Sci., 25: 163-170. DOI: $10.4314 /$ ejhs.v25i2.9

Khattab, M., Y.S. Khader, A. AI-Khawaldeh and K. Ajlouni, 2010. Factors associated with poor glycemic control among patients with Type 2 diabetes. J. Diabetes Complications, 24: 84-89. DOI: $10.1016 /$ j.jdiacomp.2008.12.008

Kyngäs, H.A., 2007. Predictors of good adherence of adolescents with diabetes (insulin-dependent diabetes mellitus). Chronic Illness, 3: 20-28. DOI: $10.1177 / 1084822311414792$

Lawrence, W., S. Conrad and A.S. Moore, 2012. Type 2 diabetes: Growing to epic proportions. Nursing Manage., 43: 20-25.

Leon, N., R. Surender, K. Bobrow, J. Muller and A. Farmer, 2015. Improving treatment adherence for blood pressure lowering via mobile phone SMSmessages in South Africa: A qualitative evaluation of the SMS-text Adherence SuppoRt (StAR) trial. BMC Family Practice, 16: 80-80. DOI: $10.1186 / \mathrm{s} 12875-015-0289-7$

Melko, C.N., P.E. Terry, M.S. Camp and M.L. Healy, 2010. Diabetes health coaching improves medication adherence: A pilot study. J. Lifestyle Med., 4: 187-193. DOI: $10.1177 / 1559827609351131$

Midhet, F.M. and A.A. Al-Mohaimeed, 2010. Lifestyle related risk factors of type 2 diabetes mellitus in Saudi Arabia. Saudi Med. J., 31: 768-774.

$\mathrm{MOH}, 2014$. Minister of health announces the results of the national survey for the health information in the Kingdom. MOH.

Polit, D.F. and C.T. Beck, 2012. Nursing Research: Generating and Assessing Evidence for Nursing Practice. 1st Edn., Lippincott Williams and Wilkins, Philadelphia, ISBN-10: 0781794684, pp: 796.

Rimer, B.K. and K. Glanz, 2005. Theory at a Glance: A Guide for Health Promotion Practice. 1st Edn., US Department of Health and Human Services, Bethesda, pp: 48.

Saeed, A.A., N.A. Al-Hamdan, A.A. Bahnassy, A.M. Abdalla and M.A.F. Abbas et al., 2011. Prevalence, awareness, treatment and control of hypertension among Saudi adult population: A national survey. Int. J. Hypertension. DOI:10.4061/2011/174135 
Saleh, F., S.J. Mumu, A. Hafez and L. Ali, 2014. Nonadherence to self-care practices and medication and health related quality of life among patients with type 2 diabetes: A cross-sectional study. BMC Public Health, 14: 431-431. DOI: 10.1186/1471-2458-14-431

Smeltzer, S.C., B.G. Bare, J.L. Hinkle and K.H. Cheever, 2010. Brunner and Suddarth's Textbook of Medical-Surgical Nursing. 1st Edn., Lippincott Williams and Wilkins, Philadelphia, ISBN-10: 0781785898 , pp: 2240.

Tiv, M., J.F. Viel, F. Mauny, E. Eschwège and A. Weill et al., 2012. Medication Adherence in Type 2 Diabetes: The ENTRED study 2007, a French population-based study. PLoS ONE.

DOI: 10.1371/journal.pone. 0032412
WHO, 2011a. Non-communicable diseases country profiles. World Health Organization.

WHO, 2011b. Global Status Report on Noncommunicable Diseases 2010. 1st Edn., World Health Organization, Geneva, ISBN-10: 9241564229, pp: 162.

WHO, 2014. Global Health Statistics. 1st Edn., Ice Press, Geneva, ISBN-10: 9240692673, pp: 180.

Wong, M.C., A.P. Kong, W.Y. So, J.Y. Jiang and J.C. Chan et al., 2011. Adherence to oral hypoglycemic agents in 26782 Chinese patients: a cohort study. J. Clin. Pharmacol., 51: 1474-1482. DOI: $10.1177 / 0091270010382911$ 